\title{
Habilidades SOCIAIS E PROBLEMAS DE COMPORTAMENTO DE ALUNOS COM DEFICIÊNCIA MENTAL, ALTO E BAIXO DESEM PENHO ACADÊMICO ${ }^{1}$ \author{
SOCIAL SKILLS AND BEHAVIOR PROBLEMS IN STUDENTS WITH MENTAL
} RETARDATION, HIGH AND LOW ACADEMIC PERFORMANCE
}

\author{
Andréa Regina Rosin-PIN OLA² \\ Zilda A parecida Pereira DEL PRETTE ${ }^{3}$ \\ Almir DEL PRETTE ${ }^{3}$
}

\begin{abstract}
RESU M O : as habilidades sociais vêm sendo amplamentereconhecidas como importante componente do processo de escolarização especialmente dos alunos com deficiência mental. A revisão da literatura evidencia escassez de pesquisas que focalizam a inclusão desses alunos no sistema regular de ensino. Considerando tal situação, este estudo teve como objetivos: avaliar e comparar o desempenho social (habilidades sociais e problemas de comportamento) e acadêmico de alunos, com deficiência mental, incluídos, em relação a seus colegas de alto e baixo rendimento acadêmico ( $A R$ e $B R$, respectivamente). Trinta professores avaliaram 120 al unos (40 com deficiência mental incluídos no ensino regular, $40 \mathrm{AR}$ e $40 \mathrm{AR}$ ) da préescola à oitava série por meio do Sistema de A valiação de Habilidades Sociais, na sua versão adaptada para o Brasil (SSRS-BR) por BANDEIRA et al. (s.d.). Os dados foram analisados estatisticamente e os resultados mostraram que: no desempenho acadêmico, os três grupos diferenciaram-se significativamente, com o AR mais positivamente avaliado, seguido pelo BR e depois pelo DM; nas habilidades sociais, AR apresentou escore acima da média normativa diferenciando de BR e DM, estes somente se diferenciaram nos fatores de A ssertividade e A utodefesa; nos problemas de comportamento, AR apresentou escore abaixo da média normativa, diferenciando-se dos dois outros grupos; quenão se diferenciaram. As evidências de dificuldades acadêmicas e interpessoais, tanto do grupo BR como DM, sugerem concomitância destas variáveis eaponta similaridades nas necessidades educativas desses dois grupos, o quereforça a importância das habilidades sociais para o sucesso e a inclusão escolar.
\end{abstract}

PALAVRAS-CHAVE: habilidades sociais; deficiência mental; inclusão; educação especial.

ABSTRACT: social skills have been widely recognized as important components of the schooling process, especially for students with mental deficiency. A review of the literature has shown scant research focusing on these students' inclusion in the regular school system. Considering this situation, the present study aimed: to evaluate social performance (social abilities and behavior problems) and academic achievements of students who were included in the regular school system, by comparing students with mental deficiency with their peers who had high and low academic achievement (HA and LA, respectively). Thirty teachers evaluated 120 students ( 40 with mental deficiency included in the regular system, 40 with high and 40 with low academic achievement) from preschool to the $8^{\text {th }}$ grade elementary level using the Social Skills Rating System, originally produced in the United States (Social Skills Rating System, SSRS, by Gresham \& Elliott, 1990) in its Brazilian version (SSRS-BR) adapted by Bandeira et al. (n.d.). After descriptive and inferential statistical analyses, the results showed that: concerning academic performance, the three groups showed significantly differences. The HA had the highest scores, followed LA and finally by MD; regarding social skills, HA scores were above the standard average, differing from LA and MD; these last two groups differed only as to the Assertiveness and Self-defense factors. Considering behavior problems, HA scores were bel ow the standard average, differing from the two other groups which showed no difference between groups. Academic and interpersonal difficulties for both groups LA and MD suggest concomitance of thesevariables, pointing to similar educational needs for both groups, which reinforces the importance of promoting social skills for success in school and for an effective inclusion process.

KEYWORDS: social skills; mental deficiency and inclusion; special education.

${ }^{1}$ Este texto é parte da Dissertação de Mestrado da primeira autora, orientada pela segunda e conduzida com bolsa FA PESP. O texto contou com a colaboração do terceiro autor e, na sua versão preliminar foi avaliado por Camila de Souza Pereira, participante do RIHS (www.rihs.ufscar.br), a quem os autores agradecem.

2 Psicóloga, Doutoranda do Programa de Pós-graduação em Psicologia da USP-Ribeirão Preto.

3 Professores Titulares do Departamento de Psicologia da Universidade Federal de São Carlos, Orientadores do Programa de Pós-graduação Educação Especial da UFSCAR e bolsistas de Produtividade em Pesquisa do CNPq (Web.-page: www.rihs.ufscar.br; E-mail: zdprette@power.ufscar.br). 


\section{INTRODUÇÃO}

A pós a década de 90 intensificou-se, na educação brasileira, o processo de inclusão de alunos com deficiência no ensino regular. Entende-se que o movimento deinclusão, não se restringe à inserção de alunos com deficiências nas escolas regulares, mas visa criar também condições necessárias para que todos os alunos tenham acesso e permaneçam neste tipo de ensino ao longo dos anos escolares (MANTOAN, 2003; MITTLER, 2003).

Considerando esse contexto, estudos (BATISTA; ENUMO, 2004; CAPELLINI, 2001; CAPELLINI, 2004; ZANATA, 2004) sugerem que a maior dificuldade ocorre para com os alunos com deficiência mental, já que estes apresentam baixo rendimento acadêmico e as grandes dificuldades de interação social.

A poiada nas definições científicas sobre a deficiência mental, como a da Associação A mericana de Retardo Mental (AAMR), a compreensão sobre essa deficiência vem se alterando nas últimas décadas, deixando, para um passado remoto, a perspectiva popular e passand o gradativamente para uma compreensão científica, comprometida com avaliações cuidadosas e sistemas de atendimentos mais eficazes.

A mais recente definição de deficiência mental da AAMR, apresentada por Luckasson et al. (2002), pode ser resumida como: [...]. uma incapacidade caracterizada por limitações significativas em ambos, funcionamento intel ectual e comportamento adaptativo, e se expressa, nas habilidades sociais, conceituais e práticas (grifo nosso).

Esta definição apresenta três indicadores do diagnóstico da deficiência mental: as habilidades conceituais e práticas, que podem ser entendidas como capacidades abstratas e de solução de problemas concretos, respectivamente, eas habilidades sociais, estas entendidas como capacidade deengajar-seem atividades sociais produtivas e duradouras. Com baseno referencial conceitual da construção social de conhecimento e sua relação com o campo teórico-prático das habilidades sociais (DEL PRETTE; DEL PRETTE, 1998), pode-se afirmar que essas três habilidades são interdependentese, nesse sentido, deveriam merecer igual atenção dos educadores comprometidos com a inclusão dos indivíduos com deficiência mental no ensino regular.

A importância das habilidades sociais vem sendo reconhecida, deforma mais generalizada, para todas as crianças, como um componente crucial para a aprendizagem e o sucesso escolar, bem como para o desenvolvimento socioemocional e o ajustamento na escola. O suporte empírico desta afirmação encontra-seem vários estudos internacionais (A GOSTIN ; BAIN , 1997; GRESHAM; ELLIOTT 1987; ELLIOT, RA CINE; BUSSE, 1995) queidentificaram como essenciais para o ajustamento social na escola, um conjunto de habilidades, definidas como acadêmicas (DEL PRETTE; DEL PRETTE, 2005) que incluem, entre outras, as de 
solicitar ajuda, fazer/ responder perguntas, seguir instruções, fazer amizades, agradecer, pedir "por favor" edizer obrigado, iniciar, manter encerrar uma conversa ou um relacionamento.

No Brasil, a avaliação do repertório social e acadêmico de alunos com deficiência mental, seja dos que freqüentam escolas especiais (KLEIJN; DEL PRETTE, 2002), seja dos que estão inseridos no ensino regular (CAPELLINI, 2001, 2004; BATISTA; ENUMO, 2004, ZANATA, 2004) evidenciam dificuldades acadêmicas e de interação social. N a pesquisa desenvolvida por Kleijn eDel Prette (2002), os professores caracterizaram o repertório destes alunos como deficitários nas habilidades de convencer colegas a se engajarem na atividade, interromper brigas, defender colegas, demonstrar polidez, lidar com elogios, expressar sentimentos positivos, cumprimentar e consolar.

Em contexto de inclusão, os resultados da pesquisa desenvolvida por Capellini (2001) indicaram que o grupo com deficiência mental apresentou uma maior homogeneidade no desempenho acadêmico (ainda que abaixo da média) e, em relação aos comportamentos sociais, maior dificuldade de interação quando comparados com os demais com necessidades educacionais especiais. Esta dificuldade também foi verificada por Batista e Enumo (2004), ao constatarem que esses alunos permaneciam sozinhos durante a maior parte do recreio e apresentavam dificuldades de iniciar, manter e encerrar contatos sociais com colegas.

As pesquisas referidas têm apontado, no geral, para uma dificuldade no processo de inclusão de alunos com deficiência mental, seja por apresentarem um desempenho acadêmico muito abaixo da média, seja por apresentarem dificuldades deinteração social. Del PretteeDel Prette(2005) afirmaram que, apesar de não estar funcionalmente estabelecida uma relação direta entre déficits de habilidades sociais e baixo rendimento acadêmico, as pesquisa vêm trazendo evidências sobre a associação entre essas variáveis. Em relação às políticas de inclusão, defendem o desenvolvimento interpessoal, seja de alunos ou professores, é componente indispensável desse processo.

Quanto às dificuldades de aprendizagem (evidenciada pelo baixo rendimento acadêmico), as pesquisas têm evidenciado sua concomitância com as dificuldades interpessoais, expressas em termos de déficits de habilidades sociais eocorrência de problemas de comportamento. No estudo deStevanato et al . (2003) verificou-se que crianças com dificuldades de aprendizagem apresentavam dificuldades sociais e familiares (sendo avaliadas como menos populares do que as crianças sem dificuldades) e autoconceito comprometido em termos de pensamento e afetividade. Del Prette et al. (1998) constataram que essas crianças foram mais negativamente avaliadas pelos professores em termos de interações com seus pares, maior agressividadee baixa assertividade. Em outro estudo, (DEL PRETTE; DEL PRETTE, 2003) evidenciou-se comprometimento na auto-avaliação e autoconceito destas crianças: elas se auto-avaliaram de forma menos positiva, 
como menos queridas pel os colegas, menos alegres, menos colaboradoras, menos espertas e menos queridas pel os professores.

Um agravante adicional para os déficits de habilidades sociais, é que estes estão, geralmente, associados a diferentes tipos de problemas de comportamento, o que compromete a adaptação e o rendimento acadêmico na escola (LINHARES et al., 1993; MARTINI; BUROCH OVITCH,1999).

Com basena literatura dePsicopatologia Infantil, Del Prettee Del Prette (2005) definem os dois grandes grupos de problemas decomportamento na infância em:: internalizantes, que se expressam predominantemente em relação ao próprio indivíduo e podem ser caracterizados, por exemplo, por retraimento e timidez, reduzindo a chance da pessoa se expor as diversas situações de aprendizagem; e external izantes, que interferem no ambientee envolvem reações negativas em relação a outras pessoas, como por exemplo, comportamentos de agressão física, brigas, discussões, que podem dificultar o aprendizado de habilidades mais competentes egerar rejeição de pares eadultos.

Esse conjunto de evidências de pesquisa permite supor que o comprometimento interpessoal associado à deficiência mental podecausar maiores prejuízos ao desenvolvimento destes indivíduos do que o rebaixamento no desempenho acadêmico, o que constitui uma questão empírica para o presente estudo. No entanto, seja no caso de crianças com dificuldade de aprendizagem, deficiência mental ou não diagnosticadas, conforme Del Prette e Del Prette (1998; 2004; 2005), vários estudos vêm defendendo que a avaliação e na promoção do repertório social de todas as crianças pode contribuir para o planejamento de estratégias de promoção do desenvolvimento eaprendizagem em contexto escolar, e que a prevenção além de eficaz, resulta em uma notável economia ao país.

sse investimento significa criar condições escolares para o desenvolvimento socioemocional das crianças, o que pode favorecer tanto as crianças com dificuldade de aprendizagem e deficiência mental incluídas, como todas as demais (DEL PRETTE; DEL PRETTE, 2001; DEL PRETTE; DEL PRETTE, 2005; DEL PRETTE et al., 1998; KLEIJN; DEL PRETTE, 2002).

Considerando a atual preocupação com a inclusão de crianças com necessidades educacionais especiais no ensino regular, pareceimportanteidentificar os déficitse recursos interpessoais decrianças, com esem deficiência ou dificuldades deaprendizagem, para mel hor planejamento desse processo. Esteestudo tevecomo objetivos: 1) avaliar o desempenho social (habilidades sociais e problemas de comportamento) deuma amostra heterogênea dealunos (igual proporção decrianças com deficiência mental, baixo e al to rendimento acadêmico) e 2) compará-los a fim deidentificar possíveis déficits quepoderiam estar dificultando o processo deind usão dos alunos com deficiência mental. A comparação com alunos de baixo e alto rendimento acadêmico foi planejada como forma de isolar o fator acadêmico das outras possíveis variáveis associadas à deficiência mental. 


\section{Metodologia}

\subsection{Participantes}

O presente estudo foi aprovado pelo Comitê de Ética (Protocolo no. 038/ 04 da CEP/ UFSCar) e a coleta de dados ocorreu conforme as prescrições detal hadas naquele documento.

A amostra foi composta por 30 professores que avaliaram 120 alunos organizados em três grupos: 40 com deficiência mental (DM) incluídos na rede regular de ensino, 40 indicados pelos professores como de alto rendimento acadêmico (AR) e 40 de baixo rendimento (BR). A amostra decrianças provinha de 30 salas de aula, de préescola à 8a. série, sendo 59 meninos (49,2\%) e 61 meninas $(50,8 \%)$, idade variando de 4 a 19 anos $(M=9 ; d p=3,13)$ e nível sócio-econômico entre $A 1$ e D (com maioria de C), conforme classificação do Critério Brasil (www.ebap.org). Todas as crianças com DM possuíam al gum tipo de documento oficial quecomprovava o diagnóstico e, no Testede Raven, obtiveram escore abaixo do percentil 25. Dos 30 professores, somente dois eram do sexo masculino, com idade variando entre 22 a 60 anos (Média=36; $d p=11,12$ ) e tempo de magistério entre 3 e 2lanos (Média=8; e dp=1,68).

\subsection{LOCAIS DE COLETA DE DADOS}

Esteestud o foi desenvolvido em noveescolas (duas particulares esetemunicipais) localizadas em diferentes regiões (centro e periferia) de uma cidade com aproximadamente 650 mil habitantes, do interior de São Paulo. As escolas foram as quetinham alunos com deficiência mental incluídos, segundo dados da Secretaria Municipal da Educação.

\subsection{NSTRUMENTOS}

Q uestionário Critério Brasil (ww w .ebap.org). Instrumento quemede o poder aquisitivo do consumidor conforme os critérios estabelecidos pela Associação Brasileira de A nunciantes (ABA) eA ssociação N acional das Empresas de Pesquisa de Mercado (ANEP), com a participação da Associação Brasileira dos Institutos de Pesquisa de Mercado (ABIPEME), com base nos levantamentos socioeconômi cos de 1993 a 2000. A posição socioeconômica é baseada na posse de bens de consumo duráveis, instrução do chefe da família e outros fatores, como a presença de empregados domésticos para caracterização dos participantes, produzindo uma classificação estratificada em cinco classes (A 1, A2, B1, B2, C, D, E) sendo que as duas de mai or poder aquisitivo foram subdivididas.

Teste de Raven - M atrizes Coloridas (ANGELINI et al.,1987). Avalia o nível intelectual de crianças de 5 a 14 anos, sendo possível aplicar em idades superiores, caso a pessoa tenha deficiência mental. A faixa de percentil entre 0-25 caracteriza os al unos como "intelectual mente deficientes". 
Sistema de A valiação de Habilidades Sociais (Social Skills Rating System, - SSRS, originalmente produzido nos Estados Unidos por GRESHAM; ELLIOTT, 1990), em versão adaptada pra o Brasil por Bandeira et al. (SSRS-BR). Conjunto de formulários que permitem avaliar habilidades sociais, problemas de comportamento e desempenho acadêmi co e podem ser usados com alunos de pré escola e ensino fundamental. Os formulários são apresentados em três versões: para auto-avaliação do aluno, para pais e para professores. N este estudo somente foi utilizado o formulário para professores, sob a forma de entrevista. O professor avalia cada aluno em termos da freqüência de emissão de habilidades sociais (30 itens), de comportamentos problemáticos (18 itens) e de desempenho acadêmico (nove itens), bem como a importância dos itens de habilidades sociais. As escalas de freqüência e importância são respondidas com base em três pontos (nunca, al gumas vezes e muito freqü entemente, no primeiro caso, e não importante, importante e indispensável, no segundo). Na escala de competência acadêmica, o professor compara o aluno com as outras crianças da sala, utilizando uma escala de cinco pontos (entre os $10 \%$ piores, $20 \%$ piores, $40 \%$ médios, $20 \%$ bons ou entre os $10 \%$ ótimos) em leitura, matemática, motivação geral, participação dos pais, funcionamento intel ectual e comportamento geral em classe. No estudo de validação nacional (BANDEIRA et al., s.d), identificou-se uma estrutura fatorial para habilidades sociais com cinco fatores queexplicaram $62,04 \%$ da variância equeforam nomeados como: Fator 1- Responsabilidade/ Cooperação (alfa=0,93, com 15 itens); Fator 2A sserção (alfa $=0,89$, com nove itens); Fator 3- A utocontrole (alfa $=0,88$, com nove itens); Fator 4- Autodefesa (alfa $=0,80$, com três itens); Fator 5- Cooperação com pares (alfa $=0,74$, com quatro itens). A estrutura fatorial da escala de comportamentos problemáticos produziu dois fatores que explicaram 55,78\% da variância: Fator 1-Comportamentos External izantes (alfa=0,93, com 13 itens); Fator 2-Comportamentos Internalizantes (alfa $=0,74$, com seis itens, sendo que o item 38 , apareceu nos dois fatores).

\subsection{Procedimento de coleta de dados}

A pós os trâmites éticos iniciais, foi realizada a coleta de informações sobresexo, idadeehistórico deescolarização dos alunos. Em seguida, os professores responderam o SSRS-BR - individual mente e na presença da pesquisadora - para cada uma das crianças, indicando a freqüência de resposta apresentada pelo aluno, em cada item de habilidades sociais e de comportamentos problemáticos; a importância de cada item de habilidades sociais e a classificação da criança nos itens de competência acadêmica.

\section{RESULtAdos}

As avaliações dos professores foram computadas em escores individuais (gerais e fatoriais) de freqüência de habilidades sociais, problemas de comportamento, utilizando-se a estrutura fatorial nacional já referida. Foram 
realizadas as análises estatísticas descritivas decada um dos grupos (média, desvio padrão, erro padrão da média) nos escores obtidos e a comparação entre os três grupos entresi ecom a referência normativa brasileira (BANDEIRA et al., s.d.) por meio de ANOVA (com post hoc de TUKEY para comparação dos grupos dois a dois), a fim de identificar habilidades sociais que poderiam ser deficitárias em cada grupo.

A Figura 1 apresenta os resultados dos grupos AR (alto rendimento), BR (baixo rendimento) e RN (referência normativa) em relação ao desempenho acadêmico global.

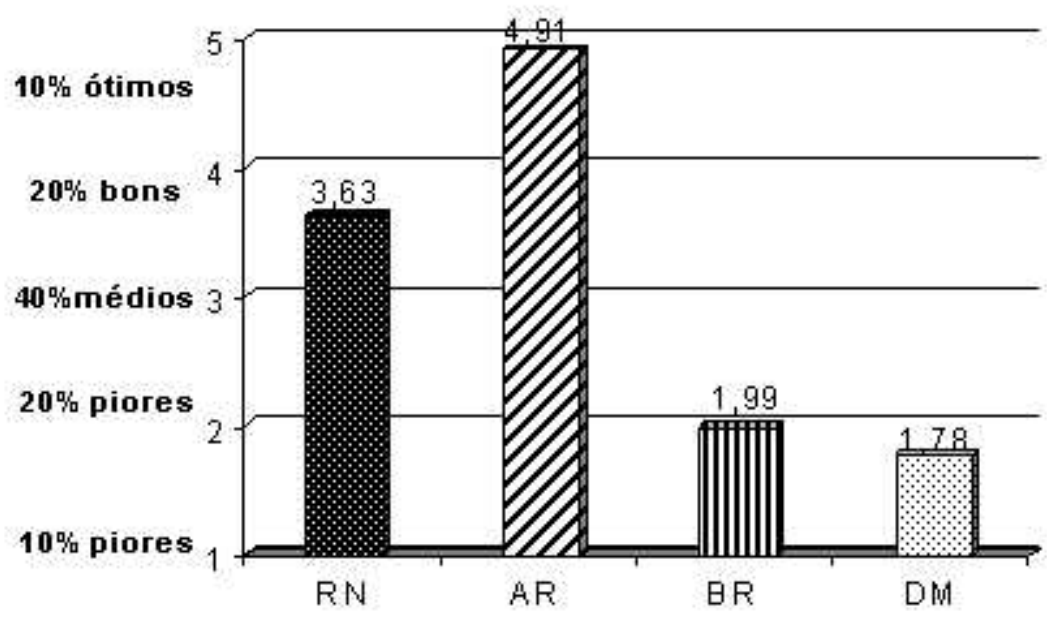

Figura 1 - Valores de desempenho acadêmico global dos grupos (RN- Referência N acional, AR-A Ito Rendimento, BR- Baixo Rendimento e DM- Deficiente Mental)

A Figura 1 mostra que o desempenho acadêmico do grupo AR foi avaliado com pontuação bem próxima à máxima que seria 5 (entre os $10 \%$ ótimos da sala) e superior à da referência normativa; já o grupo BR, próximo a 2 (entre os $20 \%$ piores) eDM , pontuação entre 1 e 2 (entre os 10 e $20 \%$ piores)-foram inferiores, conforme esperado, à referência normativa,. Portanto, pode-se afirmar que a indicação inicial dos professores sobre os al unos que estavam entre os piores e os melhores em matemática eleitura écoerente com os dados produzidos pela escala SSRS-BR.

A o comparar o desempenho acadêmico global dos grupos, verificouseque $A R, B R$ eDM apresentaram diferenças significativas entre si $(F=541,296, p=$ 0,000), sendo que: (a) o grupo AR foi avaliado como apresentando desempenho acadêmico significativamente superior ( $p \varangle 0,00$ ) ao DM eBR; e (b) DM e BR não se diferenciaram entresi. 
A Tabela 1 apresenta os resultados dos grupos AR, DM e BR em cada um dos itens de desempenho acadêmico avaliados.

Tabela 1- Dados descritivos (média e desvio padrão) dos escores dos grupos AR (Alto Rendimento), BR (Baixo Rendimento) eDM (Deficiente Mental) nos itens de desempenho acadêmico do SSRS ( $\mathrm{N}=40$ para cada grupo).

\begin{tabular}{|c|c|c|c|c|}
\hline ITENS DO SSRS-BR & $\begin{array}{c}\text { AR } \\
\text { Media (dp) }\end{array}$ & $\begin{array}{c}\text { BR } \\
\text { Media (dp) }\end{array}$ & $\begin{array}{c}\text { DM } \\
\text { Media (dp) }\end{array}$ & DIFERENÇAS \\
\hline Item 49- Desempenho Acadêmico geral & $4,97(0,14)$ & $1,62(0,14)$ & $1,37(0,14)$ & $\mathrm{DM}=\mathrm{BR} / \mathrm{AR}>\mathrm{BR}=\mathrm{DM}^{* *}$ \\
\hline Item 50- Desempenho Leitura & $5,00(0,12)$ & $1,70(0,12)$ & $1,20(0,12)$ & $\mathrm{AR}>\mathrm{BR}>\mathrm{DM} * *$ \\
\hline Item 51- Desempenho Matemática & $4,95(0,15)$ & $1,77(0,15)$ & $1,22(0,15)$ & $\mathrm{AR}>\mathrm{BR}>\mathrm{DM}^{* *}$ \\
\hline Item 52- Expectativa Leitura & $4,85(0,14)$ & $1,85(0,14)$ & $1,40(0,14)$ & $\mathrm{AR}>\mathrm{BR}>\mathrm{DM} * *$ \\
\hline Item 53- Expectativa Matemática & $4,85(0,16)$ & $1,95(0,16)$ & $1,32(0,16)$ & $\mathrm{AR}>\mathrm{BR}>\mathrm{DM} * *$ \\
\hline Item 54- Motivação geral & $4,92(0,20)$ & $2,15(0,20)$ & $2,37(0,20)$ & $\mathrm{DM}=\mathrm{BR} / \mathrm{AR}>\mathrm{BR}=\mathrm{DM}^{* *}$ \\
\hline Item 55- Estímulo dos pais & $4,85(0,23)$ & $2,25(0,23)$ & $2,80(0,23)$ & $\mathrm{DM}>\mathrm{BR} * / \mathrm{AR}>\mathrm{DM}>\mathrm{BR} * *$ \\
\hline Item 56- Funcionamento intelectual & $4,92(0,16)$ & $2,05(0,16)$ & $1,60(0,16)$ & $\mathrm{AR}>\mathrm{BR}>\mathrm{DM}^{* *}$ \\
\hline Item 57-Comportamento Geral & $4,87(0,24)$ & $2,57(0,24)$ & $2,80(0,24)$ & $\mathrm{DM}>\mathrm{BR} * / \mathrm{AR}>\mathrm{DM}>\mathrm{BR} * *$ \\
\hline
\end{tabular}

$(* p<05 ; * p<01)$

N esta tabela é possível perceber que: (a) o grupo AR foi avaliado com média maior esignificativamentediferente dos grupos BR eDM em todos os itens; (b) os grupos BR eDM se diferenciaram nos itens espećficos deleitura ematemática e no funcionamento intelectual (item 50, 51, 52, 53 e 56), com desvantagem para o grupo DM, e nos itens 55 e 57, com desvantagem para o grupo BR; (c) os grupos DM e BR assemel haram-se nos itens 49 e 54.

Observa-se, ainda, que os grupos BR e DM, por mais que tenham se diferenciado nos itens de específicos de leitura ematemática, foram avaliados com média inferior a 2, situando-se, na classificação do SSRS-BR, entre os 10\% e 20\% piores alunos da classe. Nos itens 54, 55 e 57, o grupo DM foi avaliado um pouco melhor que nos demais itens, com média entre 2 e 3 (entre os $20 \%$ piores e os $40 \%$ médios) ecom escoresuperior ao do grupo BR. Este dado evidencia as dificuldades acadêmicas dos grupos BR eDM. Nas habilidades específicas deleitura, matemática e funcionamento intelectual, o grupo com deficiência mental foi avaliado mais negativamente, mesmo dispondo de maior "Estímulo dos pais" e pontuação mais alta no item Comportamento em sala.

A Figura 2 apresenta o resultado dos grupos AR, BR eDM nos fatores de habilidades sociais do SSRS-BR, comparados com os dados normativos (RN). 


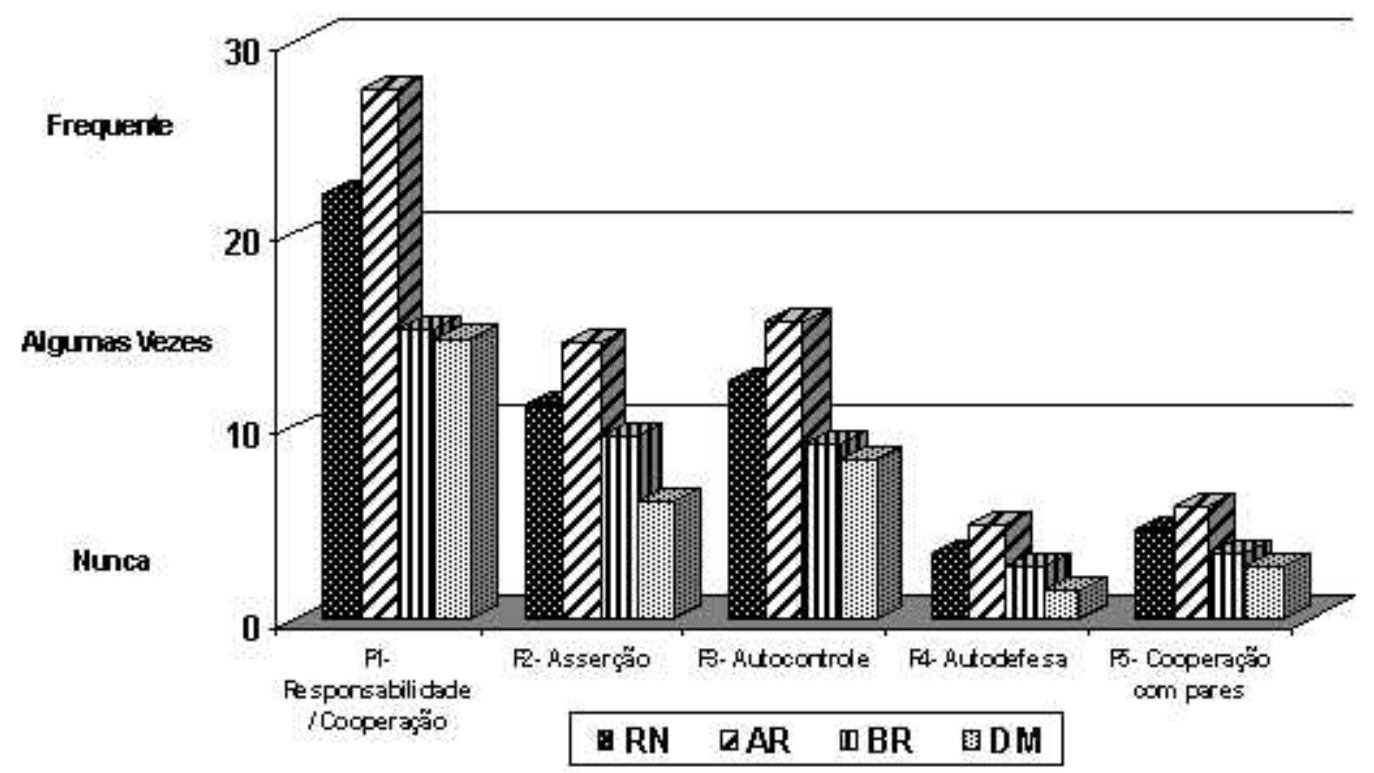

Figura 2 - Comparação dos dados da Referência Nacional (RN) com os dados dos grupos DM/ AR/ BR nos fatores de habilidades sociais do SSRS-BR.

N ovamente, evidencia-seo padrão superior einferior, respectivamente, dos grupos AR e BR, em relação à amostra normativa (RN). Verifica-se também, que os grupos BR e DM apresentaram escores inferiores aos do grupo RN, em todos os fatores de habilidades sociais.

A Tabela 2 mostra as diferenças entre os grupos (comparados dois a dois) nos fatores de habilidades sociais.

Tabel a 2 - Resultados da anál ise comparativa entre os grupos nos escores fatoriais de habilidades sociais do SSRS-BR ( $N=40)$.

\begin{tabular}{|c|c|c|c|c|}
\hline FATORES DO SSRS-BR & $\begin{array}{c}\text { AR } \\
\text { Media (dp) }\end{array}$ & $\begin{array}{c}\text { BR } \\
\text { Media (dp) }\end{array}$ & $\begin{array}{c}\text { DM } \\
\text { Media (dp) }\end{array}$ & DIFERENÇAS \\
\hline$\overline{\text { F1- Responsabilidade/Cooperação }}$ & $27,425(2,58)$ & $15,025(5,94)$ & $14,525(6,71)$ & $\mathrm{DM}=\mathrm{BR} / \mathrm{AR}>\mathrm{BR}=\mathrm{DM}^{* *}$ \\
\hline F2- Asserção & $14,375(2,77)$ & $9,125(4,10)$ & $6,125(4,39)$ & $\mathrm{AR}>\mathrm{BR}>\mathrm{DM}^{* *}$ \\
\hline F3- Autocontrole & $15,450(1,85)$ & $9,125(4,09)$ & $8,230(4,37)$ & $\mathrm{DM}=\mathrm{BR} / \mathrm{AR}>\mathrm{BR}=\mathrm{DM}^{* *}$ \\
\hline F4- Autodefesa & $4,875(1,38)$ & $2,641(1,67)$ & $1,425(1,56)$ & $\mathrm{AR}>\mathrm{BR}>\mathrm{DM}^{* *}$ \\
\hline F5- Cooperação com pares & $5,725(1,76)$ & $3,275(1,94)$ & $2,775(1,79)$ & $\mathrm{DM}=\mathrm{BR} / \mathrm{AR}>\mathrm{BR}=\mathrm{DM}^{* *}$ \\
\hline
\end{tabular}

$(* * p<01)$

Observa-se, nesta tabela, que: (a) os grupos BR eDM diferenciaram-se do grupo AR em todos os fatores de habilidades sociais, com desvantagem para DM; (b) os grupos BR e DM assemel haram-se nas habilidades sociais de Responsabilidade/C ooperação, A utocontrole eC ooperação com pares, apresentando baixo escore; e (c) BR eDM diferenciaram-senas habilidades deA ssertividade eA utodefesa, 
ROSIN-PINOLA, A. R.; DEL PRETTE, Z. A. P. ; DEL PRETTE, A.

com desvantagem para grupo DM.

A Tabela 3 apresenta os resultados dos três grupos (DM, AR e BR) nos itens de habilidades sociais obtidos no SSRS-BR.

Tabela 3 - Principais diferenças entre os três grupos nos itens de habilidades sociais do SSRS-BR $(\mathrm{N}=40)$.

\begin{tabular}{|c|c|c|c|c|c|}
\hline $\begin{array}{l}\text { Fato } \\
\text { res }\end{array}$ & Itens & $\mathrm{DM}$ & $\mathrm{BR}$ & AR & Diferenças \\
\hline \multirow{15}{*}{ F1 } & HS1- Controla irritação em conflitos com colegas. & 1,15 & 1,07 & 1,95 & $\mathrm{AR}>\mathrm{DM}=\mathrm{BR} * *$ \\
\hline & HS8 - Usa o tempo livre de maneira aceitável. & 0,92 & 0,80 & 1,90 & $\mathrm{AR}>\mathrm{DM}=\mathrm{BR} * *$ \\
\hline & HS9 - Termina as tarefas de classe no tempo & 0,70 & 0,60 & 1,97 & $\mathrm{AR}>\mathrm{DM}=\mathrm{BR} * *$ \\
\hline & HS12 - Controla irritação conflitos com adultos. & 1,05 & 1,07 & 1,85 & $\mathrm{AR}>\mathrm{DM}=\mathrm{BR} * *$ \\
\hline & HS13 - Mostra interesse em várias coisas. & 1,12 & 1,05 & 1,82 & $\mathrm{AR}>\mathrm{DM}=\mathrm{BR} * *$ \\
\hline & HS15 - Usa o tempo apropriadamente enquanto espera. & 0,65 & 0,77 & 1,77 & $\mathrm{AR}>\mathrm{DM}=\mathrm{BR} * *$ \\
\hline & HS16 - Faz corretamente as tarefas escolares. & 0,57 & 0,62 & 1,95 & $\mathrm{AR}>\mathrm{DM}=\mathrm{BR} * *$ \\
\hline & HS20 - Segue suas instruções. & 1,00 & 1,10 & 1,92 & $\mathrm{AR}>\mathrm{DM}=\mathrm{BR} * *$ \\
\hline & HS21- Guarda o material ou objetos escolares. & 1,45 & 1,52 & 1,82 & $\mathrm{AR}>\mathrm{DM}=\mathrm{BR} * *$ \\
\hline & HS22 - Coopera com colegas sem ter que pedir. & 0,80 & 0,90 & 1,65 & $\mathrm{AR}>\mathrm{DM}=\mathrm{BR} * *$ \\
\hline & HS23 - Ajuda voluntariamente os colegas nas tarefas & 0,30 & 0,55 & 1,47 & $\mathrm{AR}>\mathrm{DM}=\mathrm{BR} * *$ \\
\hline & HS27 - Mantém a carteira limpa e arrumada & 1,00 & 1,22 & 1,80 & $\mathrm{AR}>\mathrm{DM}=\mathrm{BR} * *$ \\
\hline & HS28 - Presta atenção às suas instruções. & 1,30 & 1,12 & 1,87 & $\mathrm{AR}>\mathrm{DM}=\mathrm{BR} * *$ \\
\hline & HS29 - Muda facilmente de uma atividade para outra & 1,15 & 1,27 & 1,90 & $\mathrm{AR}>\mathrm{DM}=\mathrm{BR} * *$ \\
\hline & HS30 - Se dá bem com as pessoas diferentes. & 1,35 & 1,32 & 1,85 & $\mathrm{AR}>\mathrm{DM}=\mathrm{BR} * *$ \\
\hline \multirow{9}{*}{$\mathrm{F} 2$} & HS2 - Apresenta-se a novas pessoas sem precisar mandar. & 0,70 & 1,05 & 1,35 & $\begin{array}{l}\mathrm{DM}=\mathrm{BR} / \mathrm{BR}=\mathrm{AR} / \mathrm{AR}>\mathrm{D} \\
\mathrm{M} * *\end{array}$ \\
\hline & HS3 - Questiona apropriadamente as regras injustas. & 0,37 & 0,92 & 1,55 & $\mathrm{AR}>\mathrm{BR}>\mathrm{DM} * *$ \\
\hline & HS6 - Diz coisas boas sobre si mesmo ... & 0,67 & 0,85 & 1,72 & $\mathrm{AR}>\mathrm{DM}=\mathrm{BR} * *$ \\
\hline & HS7 - Convida outros para juntar-se em atividades. & 0,55 & 1,15 & 1,72 & $\mathrm{AR}>\mathrm{BR}>\mathrm{DM} * *$ \\
\hline & HS10 - Faz amigos facilmente. & 1,00 & 1,42 & 1,80 & $\mathrm{AR}=\mathrm{BR}>\mathrm{DM} * *$ \\
\hline & HS14 - Inicia conversação com os colegas. & 0,92 & 1,52 & 1,80 & $\mathrm{AR}=\mathrm{BR}>\mathrm{DM} * *$ \\
\hline & HS19 - Elogia os colegas. & 0,45 & 0,55 & 1,40 & $\mathrm{AR}>\mathrm{BR}>\mathrm{DM} * *$ \\
\hline & HS22 - Coopera com colegas sem ter que pedir. & 0,80 & 0,90 & 1,65 & $\mathrm{AR}>\mathrm{BR}>\mathrm{DM}^{* *}$ \\
\hline & $\begin{array}{l}\text { HS24 - Junta-se a grupo ou atividade em curso sem lh } \\
\text { pedir. }\end{array}$ & 0,65 & 1,05 & 1,37 & $\begin{array}{l}\mathrm{DM}=\mathrm{BR} / \mathrm{BR}=\mathrm{AR} / \mathrm{AR}>\mathrm{D} \\
\mathrm{M}^{* *}\end{array}$ \\
\hline \multirow{9}{*}{$\mathrm{F} 3$} & HS1 - Controla irritação em conflitos com colegas. & 1,15 & 1,07 & 1,95 & $\mathrm{AR}>\mathrm{DM}=\mathrm{BR} * *$ \\
\hline & HS4 - Negocia em conflito mudando de idéia acordo. & 0,62 & 1,07 & 1,67 & $\mathrm{AR}>\mathrm{BR}>\mathrm{DM} * *$ \\
\hline & HS5 - Reage de forma apropriada à pressão colegas. & 0,92 & 0,97 & 1,82 & $\mathrm{AR}>\mathrm{DM}=\mathrm{BR} * *$ \\
\hline & HS8 - Usa o tempo livre de maneira aceitável. & 0,92 & 0,80 & 1,90 & $\mathrm{AR}>\mathrm{DM}=\mathrm{BR} * *$ \\
\hline & HS11 - Responde apropriadamente a gozações & 0,47 & 0,75 & 1,50 & $\mathrm{AR}>\mathrm{DM}=\mathrm{BR} * *$ \\
\hline & HS12 - Controla irritação em conflitos com adultos. & 1,05 & 1,07 & 1,85 & $\mathrm{AR}>\mathrm{DM}=\mathrm{BR} * *$ \\
\hline & HS18 - Aceita idéias dos colegas em atividades grupais. & 0,95 & 1,27 & 1,47 & $\begin{array}{l}\mathrm{DM}=\mathrm{BR} / \mathrm{BR}=\mathrm{AR} / \mathrm{AR}>\mathrm{D} \\
\mathrm{M} * *\end{array}$ \\
\hline & HS25-Responde apropriadamente quando provocado & 0,80 & 0,77 & 1,52 & $\mathrm{AR}>\mathrm{DM}=\mathrm{BR} * *$ \\
\hline & HS30 - Se dá bem com as pessoas diferentes. & 1,35 & 1,32 & 1,85 & $\mathrm{AR}>\mathrm{DM}=\mathrm{BR} * *$ \\
\hline \multirow[b]{3}{*}{ F4 } & HS3 - Questiona de forma apropriada regras injustas & 0,37 & 0,92 & 1,55 & $\mathrm{AR}>\mathrm{BR}>\mathrm{DM} * *$ \\
\hline & HS6 - Diz coisas boas sobre si mesmo & 0,67 & 0,85 & 1,72 & $\mathrm{AR}>\mathrm{DM}=\mathrm{BR} * *$ \\
\hline & HS17 - Argumenta apropriadamente tratado injusta... & 0,37 & 0,85 & 1,60 & $\mathrm{AR}>\mathrm{BR}>\mathrm{DM} * *$ \\
\hline \multirow{4}{*}{ F5 } & HS22 - Coopera com colegas sem ter que pedir. & 0,80 & 0,90 & 1,65 & $\mathrm{AR}>\mathrm{DM}=\mathrm{BR} * *$ \\
\hline & HS23 - Ajuda voluntariamente os colegas nas tarefas & 0,30 & 0,55 & 1,47 & $\mathrm{AR}>\mathrm{DM}=\mathrm{BR} * *$ \\
\hline & $\begin{array}{l}\text { HS24 - Junta-se a grupo ou atividade em curso sem lhe } \\
\text { pedir. }\end{array}$ & 0,65 & 1,05 & 1,37 & $\begin{array}{l}\mathrm{DM}=\mathrm{BR} / \mathrm{BR}=\mathrm{AR} / \mathrm{AR}>\mathrm{D} \\
\mathrm{M}^{* *}\end{array}$ \\
\hline & HS26 - Ignora distrações dos colegas nas tarefas de classe.. & 1,02 & 0,77 & 1,22 & $\begin{array}{l}\mathrm{DM}=\mathrm{BR} / \mathrm{DM}=\mathrm{AR} / \mathrm{AR}>\mathrm{B} \\
\mathrm{R}^{* *}\end{array}$ \\
\hline
\end{tabular}

$(* * p<01)$ 
Com a Tabela 3, observa-se: (a) um padrão geral de superioridade do grupo AR sobre BR e DM, que se alterou somente em seis itens: BR igualou-se com AR nos itens 10, 14, 18, 24 e 30, e no item 26, DM igualou-se a AR; (b) na maioria dos itens, os grupos DM eBR não se diferenciaram eambos apresentaram baixos escores de habilidades sociais e (c) em oito itens (itens 3, 4, 7, 10, 14, 17, 19 22), o grupo BR apresentou valor médio significativamente maior que DM.

Verifica-se até aqui que, na visão do professor, o grupo AR apresenta alto escore em todas as habilidades sociais avaliadas e todos os recursos sociais necessários para seu processo deescolarização. Na avaliação do professor, o grupo $B R$, com repertório inferior a AR e superior a DM, é capaz, diferentemente do grupo DM, de defender seus próprios direitos, questionando regras injustas e argumentando quando tratado injustamentee, também, conseguemanter relações interpessoais com colegas e professores, com al guma freqüência, convidando outros colegas para juntar-se em grupo, elogiando os colegas, cooperando com estes, aceitando as idéias do grupo e negociando para chegar a uma acordo,.

O grupo DM, do ponto de vista do professor, apresenta maior comprometimento em habilidades requeridas pelo ambiente escolar (AGOSTIN; BAIN , 1997; GRESHAM ; ELLIOTT 1987; ELLIOT, RACINE, BUSSE, 1995): aúnica habilidade em que se assemel hou a AR foi a de ignorar distrações dos colegas nas tarefas declasse. A lém disso, estegrupo foi avaliado como apresentando al to escore de problemas de comportamento, semelhante ao grupo BR, o que também compromete a aquisi ção e desempenho de habilidades sociais segundo Del Prette e Del Prette(2005).

A Figura 3 apresenta os resultados dos grupos AR, BR eDM em relação aos problemas de comportamento, com indicação dos valores de RN.

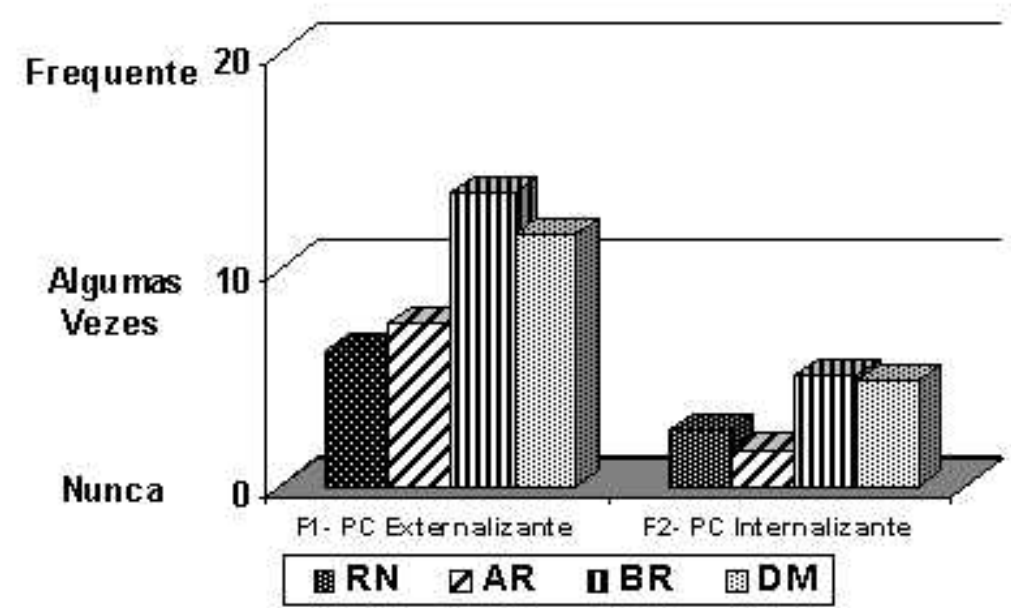

Figura 3- Comparação dos dados de Referência Nacional, com os dados dos grupos DM , AR e BR nos fatores de problemas de comportamento. 
Esta Figura mostra que: (a) os dados do grupo AR estão mais próximos da referência normativa do que os demais grupos e (b) os grupos BR e DM apresentam significativamente mai ores escores de problemas de comportamento do que o grupo AR eRN.

NaTabela 4, são apresentados os resultados dos grupos nos indicadores de problemas de comportamento.

Tabela 4 - Resultados da análise comparativa entre os grupos nos fatores de Problemas de Comportamento (PC) do SSRS-BR.

\begin{tabular}{lcccc}
\hline \multicolumn{1}{c}{ FATORES DO SSRS-BR } & \multicolumn{1}{c}{ AR } \\
& \multicolumn{1}{c}{$\begin{array}{c}\text { Bedia }(\mathrm{dp}) \\
\text { Media }(\mathrm{dp})\end{array}$} & $\begin{array}{c}\text { DM } \\
\text { Media (dp) }\end{array}$ & DIFERENÇAS \\
\hline F1- PC Externalizante & $0.58(0,45)$ & $1.03(0,59)$ & $0.88(0,45)$ & DM=BR>AR** \\
F2- PC Internalizante & $0.28(0.29)$ & $0.84(0.41)$ & $0.81(0,46)$ & DM=BR>AR** \\
\hline
\end{tabular}

$(* * p<01)$

Verifica-se que o grupo AR apresentou baixo escore de problemas de comportamento, diferenciando-se significativamente dos grupos BR eDM, sendo que estes não diferenciaram-se significativamente entre si nos conjuntos de problemas de comportamento internalizantes e externalizantes.

A Tabela 5 apresenta os resultados por itens de problemas de comportamento.

Tabela 5 - Principais diferenças entre os três grupos nos itens específicos de Problemas de Comportamento do SSRS-BR.

\begin{tabular}{|c|c|c|c|c|c|}
\hline Fator & Item & DM & $\overline{\mathrm{BR}}$ & AR & Diferenças \\
\hline \multirow{13}{*}{ F1 } & PC 31- Briga com os outros & 0,62 & 1,22 & 0,37 & $\mathrm{BR}>\mathrm{DM}=\mathrm{AR} * *$ \\
\hline & PC 33- Ameaça ou intimida os outros & 0,75 & 1,20 & 0,05 & $\mathrm{BR}>\mathrm{DM}=\mathrm{AR} * *$ \\
\hline & PC 35-Desconcentra-se facilmente & 1,42 & 1,55 & 0,25 & $\mathrm{DM}=\mathrm{BR}>\mathrm{AR} * *$ \\
\hline & PC 36- Interrompe a conversa dos outros & 0,67 & 0,70 & 0,25 & $\mathrm{DM}=\mathrm{BR}>\mathrm{AR} * *$ \\
\hline & PC 37 - Pertuba as atividades em andamento & 0,62 & 0,82 & 0,10 & $\mathrm{DM}=\mathrm{BR}>\mathrm{AR} * *$ \\
\hline & PC 38- Demonstra ansiedade quando em grupo & 0,65 & 0,75 & 0,25 & $\mathrm{DM}=\mathrm{BR}>\mathrm{AR} * *$ \\
\hline & PC 40- Não ouve o que os outros dizem & 0,92 & 0,95 & 0,20 & $\mathrm{DM}=\mathrm{BR}>\mathrm{AR} * *$ \\
\hline & PC 41- Discute com os outros & 0,42 & 1,05 & 0,35 & $\mathrm{DM}=\mathrm{AR}<\mathrm{BR} * *$ \\
\hline & PC 42- Retruca quando os adultos lhe corrigem & 0,50 & 0,72 & 0,20 & $\mathrm{DM}=\mathrm{AR} / \mathrm{DM}=\mathrm{BR} / \mathrm{BR}>\mathrm{AR} * *$ \\
\hline & PC 43- Fica facilmente com raiva & 0,57 & 0,87 & 0,17 & $\mathrm{DM}=\mathrm{BR}>\mathrm{AR} * *$ \\
\hline & PC 44- Tem ataques de birra & 0,72 & 0,50 & 0,05 & $\mathrm{DM}=\mathrm{BR}>\mathrm{AR} * *$ \\
\hline & PC 47- Age impulsivamente & 0,80 & 1,00 & 0,17 & $\mathrm{DM}=\mathrm{BR}>\mathrm{AR} * *$ \\
\hline & PC 48- Se mostra irrequieto ou mexe excessivamente & 0,87 & 0,87 & 0,22 & $\mathrm{DM}=\mathrm{BR}>\mathrm{AR} * *$ \\
\hline \multirow{6}{*}{$\mathrm{F} 2$} & PC 32- Tem baixa auto-estima & 0,75 & 1,20 & 0,05 & $\mathrm{BR}>\mathrm{DM}>\mathrm{AR} * *$ \\
\hline & PC 34- Parece solitário & 1,10 & 1,10 & 0,27 & $\mathrm{DM}=\mathrm{BR}>\mathrm{AR} * *$ \\
\hline & PC 38- Demonstra ansiedade quando em grupo & 0,65 & 0,75 & 0,25 & $\mathrm{DM}=\mathrm{BR}>\mathrm{AR} * *$ \\
\hline & PC 39- Fica facilmente envergonhada & 0,62 & 0,72 & 0,55 & $\mathrm{DM}=\mathrm{AR}=\mathrm{BR}$ \\
\hline & PC 45- Gosta de ficar sozinho & 0,97 & 0,65 & 0,40 & $\mathrm{DM}=\mathrm{BR} / \mathrm{BR}=\mathrm{AR} / \mathrm{DM}>\mathrm{AR} * *$ \\
\hline & PC 46- Mostra-se triste ou deprimido & 0,77 & 0,70 & 0,15 & $\mathrm{DM}=\mathrm{BR}>\mathrm{AR} * *$ \\
\hline
\end{tabular}

$(* * p<0,001)$ 
No Fator 1, problemas do tipo Externalizante: (a) o grupo AR apresentou escore significativamente menor que BR e DM, com exceção dos itens 31, 33 e 42 em que DM assemelhou-se a AR, apresentando baixo escore e (b) os grupos BR e DM assemelharam-se na maioria dos itens, com exceção dos itens 31, 33 e 42, em que o grupo DM apresentou média significativamente menor que BR.

No Fator 2, problemas do tipo Internalizante: (a) os grupos BR e DM seassemel haram em todos os itens, com exceção do item 32 em que BR apresentou escore significativamente maior, (b) BR e DM apresentaram médias significantemente superiores ao grupo $A R$, com exceção dos itens 39 em que os grupos não se diferenciaram eno item 45 que o grupo DM foi avaliado com escore superior ao do grupo AR.

\section{Discussão}

Os resultados deste estudo mostram que, na avaliação do professor sobre o desempenho acadêmico geral dos grupos de baixo desempenho acadêmico e com deficiência mental, não apresentou diferenças significativas, isto é, ambos apresentam dificuldades acadêmicas.

Estedado, somado à avaliação queo professor fez destegrupo, em relação às habilidades sociais e problemas de comportamento, que, além das dificuldades acadêmicas, as crianças DM apresentam comprometimento no repertório social que certamente impactam negativamente sobre o processo de inclusão.

Com relação às habilidades sociais, tanto o grupo de baixo desempenho acadêmico quanto o grupo com deficiência mental foram avaliados apresentando baixo escore nos fatores Responsabilidade, A utocontrole e Cooperação com pares, que reúnem habilidades tradicional mente consideradas importantes pelos professores para o contexto escolar e preditoras do sucesso acadêmico (A GOSTIN ; BAIN, 1997; DEL PRETTE; DEL PRETTE, 2005; ELLIOTT, RACINE; BUSSE, 1995; GRESHAM; ELLIOTT, 1987).

Nos itens deA ssertividadee A u todefesa, os grupos de baixo desempenho acadêmico e com deficiência mental diferenciaram-se, com vantagem para o grupo de baixo desempenho acadêmico em seis itens. Considerando, segundo Del Prette e Del Prette (2005), os conceitos de déficit de desempenho e déficit de aquisição, ao comparar esses dois grupos com a média da referência normativa, pode-se inferir que eles também se diferenciam quanto ao tipo de déficit: o grupo de baixo desempenho acadêmico traz indicativos de déficit de desempenho (desvantagem inferida com base na emissão de uma habilidade com freqüência inferior à esperada pelo ambiente), enquanto o grupo com deficiência mental apresentaria déficit de aquisição (desvantagem inferida com base na ausência da habilidade diante das demandas do ambiente). 
Estas diferenças são importantes por al guns motivos. Primeiramente, porque podem dar pistas das dificuldades encontradas por estes grupos, principalmente, para o grupo com deficiência mental, quetem sido apontado pela literatura como o grupo que apresenta maior dificuldade de ser incluído. Em segundo lugar, ao se pensar em procedimentos de intervenção, o grupo de baixo desempenho acadêmico apresenta habilidades relevantes com al guma freqüência, enquanto o grupo com deficiência mental não as apresenta, ou seja, para estegrupo énecessário não só incentivar a emissão das habilidades, mas também ensi ná-los a emití-las, com procedimentos específicos e diferenciados para isto.

N a discussão atual sobre inclusão, ao seconsiderar a definição dequem são os al unos com necessidades educacionais especiais e, os que requerem recursos educacionais diferenciados (MARCHESI, 2004), o foco tem sido naqueles que fracassam academicamente, sejam eles deficientes ou não. Parece importante repensar o ambiente escolar como um ambiente também de promoção da competência social e de habilidades sociais (DEL PRETTE; DEL PRETTE, 1998; 2005), o que pode favorecer ganhos acadêmicos (MELO, 2003; MOLINA, 2003). Essa mudança de compreensão impõe a necessidade dos educadores (professores, diretores, coordenadores) reconhecerem seus papéis enquanto agentes educativos e assumirem o ensino destas habilidades como recurso auxiliar no processo educacional, integrado aos objetivos acadêmicos, como propõem Del Prette e Del Prette (2005).

A aval iação dos professores sobreos problemas de comportamento dá suportea argumentação acima já que, para estes, os grupos com deficiência mental e de baixo desempenho acadêmico apresentam, igual mente e com alta freqüência, estes problemas, diferindo do grupo de alto desempenho acadêmico. Pode-se destacar, ainda, que o grupo de baixo desempenho acadêmico diferenciou-se do grupo com deficiência mental nos itens Briga com os outros, Tem baixa auto-estima e A meaça ou intimida os outros, apresentando maior escore que o grupo DM nesses itens. Estes resultados sinal izam que o grupo BR, além de dificuldades acadêmicas esociais, apresenta indicadores de problemas de comportamentos externalizantes, que perturbam o ambiente e que podem afetar negativamente a percepção de si mesmos (auto-estima).

Um outro dado preocupante foi a avaliação dos professores de que o grupo com deficiência mental gosta de ficar sozinho. No estudo de Batista e Enumo (2004), Também se constatou que estes alunos ficavam sozinhos no recreio e nas atividades de grupo. Pode-se questionar o que a escola tem feito para a inclusão destes al unos, se eles apresentam o mais baixo desempenho acadêmico e social e têm, ainda, dificuldades em fazer amigos e se integrarem ao grupo de colegas. Questiona-se, também, o que para o professor é dar-se bem com os diferentes (habilidade que ele avaliou como de alta freqüência para os três grupos), pois, 
quando avaliou os problemas de comportamentos, relatou que o grupo DM gosta de ficar sozinho.

\section{CONCLUSÕES}

Considerando que, no processo de inclusão, as necessidades educacionais especiais enfatizam o uso de recursos diferenciados para aqueles que apresentam problemas de aprendizagem (MARCHESI , 2004), a verificação, neste estudo, de dificuldades acadêmicas nos grupos de baixo desempenho acadêmico e com deficiência mental, traz fortes evidências de concomitância destas duas desvantagens. Essa concomitância reforça a importância da promoção de habilidades sociais para o sucesso escolar e, também, como fator de proteção contra os problemas de comportamento internal izantes e externalizantes, confirmando a literatura da área (LIN HARES et al., 1993; DEL PRETTE et al., 1998; STEVANATO et al., 2003).

Os resultados sugerem al guns questionamentos adicionais quanto à atual situação de escolarização no Brasil. Considerando que a legislação sobre inclusão tem seu princípio na Constituição de 1988 ena Declaração M undial sobre Educação para Todos (1990), e que a década de 90 foi marcada por inúmeras publicações sobre este processo, pode-se questionar quanto esse movimento tem contribuído para a mel horia da prática pedagógica e, o queas escolas estão fazendo para possibilitar a efetiva inclusão, entendida como acesso e apropriação do conhecimento por todos os indivíduos. A dificuldade deinclusão existiria somente para os alunos com deficiência mental, como afirmam os estudos? Ou a inclusão dos alunos com deficiência tem servido de "bode expiatório" para os problemas da escolarização de alunos que apresentam baixo desempenho acadêmico?

Os resultados deste estudo permitem reafirmar a necessidade de se investir em procedimentos que possi bilitem aos alunos, com deficiência ou não, adquirir repertórios sociais e acadêmicos que favoreçam o desenvolvimento interpessoal esocioemocional necessário para lidar com as demandas sociais atuais e futuras. Também permite considerar a importância da discussão sobre o papel dos professores, diretores e outros agentes educativos no desenvolvimento social e acadêmico dos alunos.

A pesar da relevância de estudos deaval iação como o aqui apresentado, também se reconhece suas limitações. Dada à necessidade de avaliações multimodais do desempenho social (DEL PRETTE; DEL PRETTE, 2005), um primeiro limite observado foi o uso de uma única fonte de dados, a avaliação feita pelo professor. Considera-se importante investigar as habilidades sociais dealunos por meio de outros significantes (pais, diretores, colegas, por exemplo) e por observação direta em diferentes situações. Além disso, há um grupo de alunos 
(aqueles queapresentam um desempenho acadêmico médio) quenão foi avaliado, podendo-se questionar se estes apresentam alguma dificuldade escolar (seja ela acadêmica ou social ). Estud os futuros poderiam investigar também o modo como os professores promovem, ou não, as habilidades sociais na escola e se eles as reconhecem como alicerces do aprendizado formal.

\section{Referências}

AGOSTIN, T, M.; BAIN, S. K. Predicting early school success with developmental and social skills screeners. Psychology in the Schools, v.34, n.3, p. 219-228, 1997.

ANGELINI, A. et al. M anual das matrizes progressivas coloridas. São Paulo: Casa do Psicólogo, 1987.

BANDEIRA, M. et al. Escala de avaliação das habilidades sociais de estudantes do ensino fundamental. SSRS-BR: Validação transcultural para o Brasil. Encaminhado para publicação, s.d.

BATISTA, M. W; ENUMO, S. R. F. Inclusão escolar e deficiência mental: análise da interação social entre companheiros. Estudos de Psicologia, v.9, n.1, p.101-111, 2004.

CAPELLINI, V. L. M. F. A inclusão de alunos com necessidades educacionais especiais em classes comuns: avaliação do rendimento acadêmico. 2001. 249f. Dissertação (M estrado em Educação Especial) - Centro de Educação e Ciências Humanas, Universidade Federal de São Carlos, São Carlos, 2001.

. A valiação das possi bilidades do ensino colaborativo no processo de inclusão escol ar do aluno com deficiên cia mental. 2004. 299f. Tese (Doutorado em Educação Especial) - Centro de Educação e Ciências Humanas, Universidade Federal de São Carlos, São Carlos, 2004.

DECLARAÇÃO MUNDIAL SOBRE A EDUCAÇÃO PARA TODOS (Aprovada pela Conferência Mundial sobre Educação para todos - satisfação das necessidades básicas de aprendizagem). Jomtiem, Tailândia, 5 a 9 de março de 1990.

DEL PRETTE, A.; DEL PRETTE, Z. A. P. Psicologia das relações interpessoais: vivências para o trabal ho em grupo. Petrópolis: Vozes, 2001.

DEL PRETTE, Z. A. P.; DEL PRETTE, A. Desenvolvimento interpessoal e educação escolar: o enfoque das habilidades sociais. Temas em Psicologia, n.6, v.3, p. 205-215, 1998.

. Habilidades sociais e dificuldades de aprendizagem: teoria e pesquisa sob umenfoquemultimodal. In: DEL PRETTE; Z. A. P.; DEL PRETTE, A. (Org.). H abilidades sociais, desenvol vimento e aprendizagem: Questões conceituais, avaliação e intervenção. Campinas: Alínea, 2003. p. 167-206. 
DEL PRETTE, Z. A. P.; DEL PRETTE, A .A valiação do repertório social de crianças com necessidades educacionais especiais. In: MENDES, E.G.; ALMEIDA; M. A.; WILLIAMS, L.C.A. (Org.), Temas em Educação Especial: avanços recentes. São Carlos: EduFSCar, 2004, p. 149-157.

. H abilidades Sociais na infância: teoria e prática. Petropólis: Vozes, 2005.

DEL PRETTE, Z. A. P. et al. Habilidades sociais do professor: um estudo de caso. Psicologia: R eflexão e Crítica, n.11, p. 611-623, 1998.

ELLIOTT, S. N.; RACINE, C. N .; BUSSE, R. T. Best practices in preschool social skills training. In: A. THOMAS, A.; GRIMES, J. (Org.). Best pratices in school psychology- III, (p. 1009-1020). Waschington DC: The N acional Association of School Psychologists, 1995.

GRESHAM, F. M.; ELLIOTT, S. N. Social skills rating system: circlepines, MN : American Guidance Service. USA, 1990.

. Social skills deficits of students: issues of definition, classification and assessment. Journal of Reading, W riting and Learning D isabilities International, n.3, v.2, p.131-148, 1987.

KLEIJN, M. L. V. B.; DEL PRETTE, Z. A. P. Habilidades sociais em al unos com retardo mental: análise denecessidades e condições. Cadernos de Educação Especial, n.20, p.31-54, 2002.

LINHARES, M. B. M. PARREIRA; V. L. C.; MARTURANO, A. N.; SANT'ANA, S. C. Caracterização dos motivos da procura de atendimento infantil em um serviço de psicopedagogia clínica. M edicina Ribeirão Preto, n. 26, v.2, p.148-160, 1993.

LUCKASSON, R. et al. Mental Retardation - Definition, Classification and Systems of Supports. 10.ed. Washington (DC): A merican A ssociation on M ental Retardation, 2002.

MANTOAN, M. T. E. Inclusão Escolar: O queé? Por quê? Como fazer? São Paulo: Moderna, 2003.

MARCHESI, A. Da linguagem da deficiência às escolas inclusivas. In COLL, C; PALÁCIOS, J.; MARCHESI, A. (Org.). D esenvolvimento psicológico e educação: necessidades educativas especiais e aprendizagem escolar. Porto Alegre: Artes Médicas, 2004, p. 15-30.

MARTINI, M.L.; BUROCHOVITCH, E. As atribuições de causalidade, o desenvolvimento infantil e o contexto escolar. Psico-U SF , n.4, v.2, p. 23-36, 1999.

MELO, M.H.S. Crianças com dificuldades de interação no ambienteescolar: Uma intervenção Multifocal. 2003. 146f. Tese (Doutorado em Psicologia) - Instituto de Psicologia, Universidade de São Paulo, São Paulo.

MITLTER, P. Educação Inclusiva: Contextos sociais. Trad. Windyz Brazão Ferreira. Porto Alegre: ARTMED, 2003.

MOLINA, R. H abilidades sociais e dificuldades de aprendizagem: Uma análise funcional. 2003. 89f. Dissertação (M estrado em Educação Especial) - Centro deEducação eCiências Humanas, Universidade Federal de São Carlos, São Carlos. 
ROSIN-PINOLA, A. R.; DEL PRETTE, Z. A. P. ; DEL PRETTE, A.

STEVANATO, I.S. et al. A utoconceito de crianças com dificuldades de aprendizagem e problemas de comportamento. Psicologia em Estudo, n.8, v.1, p. 67-76, 2003.

ZANATA, E.M. Práticas pedagógicas inclusivas para alunos surdos numa perspectiva col aborativa. 2004. 201f. Tese (Doutorado em Educação Especial) - Centro deEducação e Ciências Humanas, Universidade Federal de São Carlos, São Carlos. 